\title{
Motivación y resiliencia en el docente. Fortaleza y oportunidad para la escuela en El Salvador
}

Motivation and resilience in the teacher. Strength and opportunity for school in El Salvador

\section{RESUMEN}

En 2014, un estudio de caso realizado en un centro escolar del municipio de Mejicanos, en el área metropolitana de San Salvador, El Salvador, Centroamérica, muestra una situación que puede considerarse paradójica. En dicho estudio, se investigó acerca del impacto de la violencia que caracteriza al centro escolar en la motivación laboral docente. Los resultados muestran que los docentes se perciben a sí mismos motivados a realizar su trabajo en estas condiciones adversas; y que esto también se refleja en la percepción que sus alumnos quienes observan a sus maestros motivados e interesados en los estudiantes. De acuerdo a lo anterior, se puede decir que, además de estar motivados en la realización de su trabajo, estos docentes han logrado un grado de fortaleza que les dota de la capacidad de sobreponerse a las adversidades; 0 lo que es 10 mismo, son docentes con alguna asignación de resiliencia. Frente a estos resultados, en este artículo se propone contribuir en el fortalecimiento de la resiliencia en el docente como una oportunidad para la escuela salvadoreña de seguir adelante en su misión educativa en contextos de violencia.

Palabras clave: educación, El Salvador, motivación laboral docente, violencia, resiliencia

\section{ABSTRACT}

In 2014 a case study conducted in a school in the municipality of Mejicanos, in the metropolitan area of San Salvador, El Salvador, Central America presented paradoxical results that is the topic of this article. In this study, the impact of violence that characterizes the school in teachers' work motivation was investigated. These results show that teachers perceive themselves motivated to do their work in such conditions; and this is also reflected in the perception of students who perceive their teachers motivation and interest in students. According to the above, it can be said that, besides being motivated in carrying out their work, these teachers have achieved a degree of strength that gives them the ability to overcome adversity; or, in other words, these are teachers with some allocation of resilience. Given these results, this article seeks to contribute in strengthening resilience in teaching as an opportunity for the Salvadoran school to move forward in its educational mission in contexts of violence.

Key words: education, El Salvador, teaching motivation, violence, resilience

\footnotetext{
" Universidad Centroamericana José Simeón Cañas, San Salvador, El Salvador; m.r.flores.e@ gmail.com.
} 


\section{Contexto}

La situación de violencia en El Salvador no cesó después del enfrentamiento armado que padeció esta nación centroamericana durante doce ańos de guerra civil (1980-1992). La aparición de pandillas, producto, en parte, de la repatriación de migrantes desde Estados Unidos (EU) hacia esta nación fue la subsecuente expresión de violencia en este país (Amaya y Martínez, 2014).

La escuela se ha convertido en una víctima directa de esta violencia, que afecta no solo a sus alumnos, sino también a sus maestros. En 2014, según el conteo del Sindicato de Maestros de Educación Rural Urbana y Urbano Marginal de El Salvador (SIMEDUCO), las víctimas de la violencia fueron diez maestros muertos (Bolaños, 2014). En 2015, la cifra ascendió, ya que al menos 14 docentes fueron asesinado ese ańo (Flores, 2015). Al final del ciclo escolar 2016, fueron siete los docentes asesinados por el accionar de las pandillas (Hernández, 2016).

Además de la inseguridad e incertidumbre que viven los maestros como posibles víctimas mortales de esta espiral, los docentes también padecen y conviven con otras manifestaciones de violencia en la escuela: extorsión, amenazas, acoso de parte de las pandillas, falta de seguridad del trayecto de sus domicilios al centro escolar y otro tipo de agresiones (Flores, Mejía y Muñoz, 2014).

Según el Observatorio del Ministerio de Educación de El Salvador sobre los centros educativos públicos en 2015 (MINED), 23.77\% de los encuestados reportan que su seguridad interna está afectada por la presencia de pandillas como las Maras, por asaltos (16.70\%), robos $(14.91 \%)$, drogas $(14.28 \%)$, portación de armas blancas (7.46\%) y extorsiones (6.78\%), entre otros factores de riesgo.

El mismo informe detalla que los centros escolares afectados en la comunidad directamente por las Maras son $64.83 \%$ robos/ asaltos $(62.12 \%)$, drogas $(60.81 \%)$, portación de armas blancas/ fuego $(56.68 \%)$, extorsiones $(45.77 \%)$, violaciones $(21.14 \%)$, explotación sexual (17.23\%), además de otros que afectan negativamente al exterior de la escuela.

Paralelo a la situación de violencia en las escuelas, el docente tiene que enfrentar otra realidad: la de un sistema educativo 
nacional que no provisiona de los recursos e insumos suficientes y necesarios a los centros escolares, tanto en infraestructura e inmobiliaria como en recursos y materiales didácticos; en formación permanente y en mejores salarios y prestaciones. De acuerdo con el MINED (2015a), solo 40\% de sus escuelas se encuentra en buenas condiciones, $10 \%$ no tiene acceso a agua, mientras que $70 \%$ está dotado de equipos informáticos, aunque solo $23 \%$ tiene conexión a Internet y 17\% tiene biblioteca. En cuestiones de apoyo al docente, solo $10.5 \%$ tiene gabinete psicopedagógico para ayudar a los estudiantes (MINED, 2015b).

En 2014, un estudio de caso con enfoque cualitativo realizado en el Centro Escolar República Oriental del Uruguay, ubicado en la colonia Zacamil del municipio de Mejicanos, en el departamento de San Salvador, se propuso caracterizar el impacto de la violencia juvenil en la motivación intrínseca y extrínseca de los docentes de tercer ciclo de dicho centro de estudio; así también identificar medidas de estrategia para fortalecer la motivación intrínseca y extrínseca de estos (Flores, Mejía y Muñoz, 2014).

La estrategia de investigación utilizada en dicho estudio fue mediante técnicas e instrumentos de investigación social a docentes, personal de dirección (director y subdirectoras) y estudiantes, todos del tercer ciclo de educación básica del centro escolar señalado.

Los resultados de dicho estudio indican que la realidad del centro escolar no dista mucho de las problemáticas en la que se encuentra la escuela salvadoreña. Sin embargo, es caso conspicuo mencionar que, aun en ese contexto, los maestros del centro escolar se mantienen motivados en su labor docente.

Este trabajo tiene como objetivo analizar, precisamente, este resultado que muestra la fortaleza ante la adversidad de estos docentes y, en base a los mismos, contribuir en la construcción y fortaleza de la resiliencia en el docente frente a la violencia y a otras problemáticas que caracterizan a centros escolares con estas características. De esta manera se pretende profundizar más en la discusión de esta temática y que sea un insumo que pueda ser tomado en cuenta en el abordaje de los problemas que caracterizan a la escuela salvadoreńa frente al estigma de la violencia y el apoyo al maestro. 


\section{Violencia}

Para la Organización Mundial de la Salud (OMS, 1996), la violencia se define como: "el uso intencional de la fuerza o el poder físico, de hecho o como amenaza, contra uno mismo, otra persona o un grupo o comunidad, que cause o tenga muchas probabilidades de causar lesiones, muerte, daños psicológicos, trastornos del desarrollo o privaciones".

Lavena (citado en Guzmán y Monterrosa, 2013) expresa que "el fenómeno de la violencia escolar centra su mirada en dos grandes aspectos, a) los ataques físicos y b) transgresión o violación de las normas en la escuela".

Como resultado de la investigación citada, el personal docente identifica que en el centro escolar se dan distintas manifestaciones de violencia tales como: la agresión verbal, amenazas, bullying o acoso escolar, consumo de drogas, riesgo de pertenecer a pandillas, trato violento, violencia física, verbal, psicológica, sexual y social entre los estudiantes y hacia los maestros y maestras.

La dirección del centro escolar identifica como expresiones de violencia juvenil las siguientes manifestaciones: amenazas, asesinatos, consumo de drogas, extorsión, tráfico de drogas y violencia física.

Estas manifestaciones de violencia podrían repercutir en la percepción de seguridad que los docentes tienen en su lugar de trabajo e incidir en su motivación laboral. Sin embargo no es el caso en los resultados de este estudio, como se revisará a continuación.

\section{Motivación laboral docente: factores intrínsecos y extrínsecos}

La motivación laboral docente se define como la satisfacción que se genera en el docente al desempeñar su labor pedagógica, que lo impulsa a seguir en su empeño, realizando determinadas acciones en los procesos de enseñanza-aprendizaje y persistir en ellas para su culminación en buenos términos (Flores, Mejía y Muñoz, 2014).

Los factores que inciden en la motivación laboral docente son varios; algunas investigaciones dan cuenta de estos: el rendimiento escolar, el clima escolar dentro de los salones de clase y en la institución en general, la procedencia de los estudiantes, 
una relación satisfactoria entre el cuerpo docente y directivo del centro (Borgonovo, López y Rodríguez, 2007); el aprendizaje y la motivación del alumnado, buenas relaciones con colegas, mínima carga burocrática, estabilidad laboral, posibilidad de ejercer liderazgo, contribuir a la sociedad, desarrollo personal y profesional, apoyo y cooperación de los padres, estatus social, ambiente físico placentero y adecuados recursos didácticos, reconocimiento de logros, tiempo libre, posibilidad de ejercer creatividad y autonomía en el trabajo e ingreso económico satisfactorio, clima institucional, relaciones de afecto docentes-alumnado y formación docente y acreditación (Cruz y Perla, 2009).

Ayala, Cruz y Novoa (2011) identificaron que la baja seguridad en el sector público hace que los docentes tengan un menor nivel de satisfacción laboral docente. El nivel de violencia actual del país no es ajeno al área educativa, especialmente en aquellas instituciones educativas públicas que se encuentran en zonas de alto riesgo delincuencial, pues el nivel de violencia hacia alumnado y personal docente es alto.

Para estudiar la motivación, deben tenerse en cuenta aspectos intrínsecos y extrínsecos que pueden favorecer u obstaculizar, a manera de catalizadores, en la labor de enseñanza y en la percepción de bienestar en su lugar de trabajo (Flores, Mejía y Muñoz, 2014).

Motivación intrínseca. Para el docente motivado intrínsecamente no existen elementos externos necesarios para desarrollarse como docente; aprenden y buscan perfeccionamiento porque están interesados en la propia actividad. Despliegan su profesión centrada en orientar a los estudiantes a aprender de manera significativa y lo hacen por su propio placer, al margen de cualquier tipo de incentivos o recompensas. El docente motivado intrínsecamente por mejorar se preocupa por qué y de qué manera aprenden sus estudiantes, con el objetivo que logren mejores resultados académicos y, a largo plazo, se desenvuelvan como profesionales competentes con pensamiento crítico. Se incluyen aquellos compromisos previos que han resultado gratificantes en la misma área (Feixas, 2004).

Respecto de lo anterior, en el estudio base, la dirección y los docentes expresan satisfacción en su labor, a pesar de reconocer la precariedad en la que realizan su trabajo, en cuanto a recursos 
materiales, bajo nivel salarial, poco reconocimiento social y un contexto social comunitario y de país cargado de manifestaciones diversas de violencia en general y de violencia juvenil específicamente.

Este sentido de vocación y mística, manifiesta en las labores docentes, se hace evidente en el gusto por trabajar sin horarios específicos, jornada que se extiende, en muchas ocasiones, hasta el hogar y el fin de semana, experimentándose con los alumnos constantemente, brindando al interior de cada docente la oportunidad de ejercer un liderazgo positivo, en tanto que propicia el protagonismo del alumno en su crecimiento y desarrollo; como también en el compromiso y renovación del conocimiento en pedagogía.

Lo descrito por los docentes guarda relación con el esbozo teórico de que la motivación intrínseca implica la gratificación originada por la necesidad de obtener satisfacciones internas en las personas. En este sentido, y como reflejo de la congruencia entre lo teórico y la praxis.

Esta situación también la perciben los estudiantes. En efecto, los alumnos aprecian a sus maestros motivados a nivel intrínseco, puesto que manifiestan que a sus docentes les agrada su trabajo y que lo realizan de manera satisfactoria. Les observan como personas que brindan confianza y se encuentran interesados en que los estudiantes aprendan; así como también se interesan por sus problemas, son personas serviciales, se involucran en las actividades de los jóvenes como juegos, celebraciones, dinámicas, etc., no importando que en su trabajo corran peligros y riesgos.

Así, el alumnado observa comportamientos explícitos motivados de sus docentes, lo que da cuenta de la motivación intrínseca: autoestima laboral del docente, intereses del docente, vocación hacia la labor docente y expectativas del docente (Feixas, 2004).

Lo anterior permite evidenciar que hay un impacto favorable de la motivación intrínseca de docentes sobre la percepción del alumnado, a pesar de realizar su labor de enseñanza en condiciones adversas y en un contexto propicio a la violencia, lo que a su vez podría considerarse como un factor preventivo de la violencia juvenil en el centro escolar, en cuanto esta población adolescente puede inspirarse en el modelaje de actitudes y en la vinculación afectiva con sus profesores. 
Motivación extrínseca. Cuando los docentes se encuentran motivados extrínsecamente, llevan cabo una determinada actividad por el valor o la importancia que dan al resultado que consiguen: por los incentivos. Ejemplo de ello es cuando el docente pone en marcha la tarea con el objetivo de conseguir algún aspecto positivo (mejorar el currículo docente en vistas a la promoción) o por evitar algo peyorativo (evaluaciones negativas de los estudiantes, directores, colegas). Lo relevante no es el proceso o el producto, sino las consecuencias del producto: obtener una recompensa o evitar un castigo o fracaso (Feixas, 2004).

En el estudio citado, los docentes manifestaron que faltan recursos o incentivo social. En efecto, se da una serie de carencias y dificultades para ejercer la docencia en su lugar de trabajo. Estas son: la escasez de estímulos en el entorno, para motivar y orientar a conseguir metas mediante una acción específica y minar esta motivación mediante la exposición a la violencia, el poco apoyo institucional (del centro escolar y del MINED) hacia los docentes; la escasez de recursos materiales para desempeńarse; las bajos salarios y escasos incentivos sociales y de reconocimiento; el bajo rendimiento académico y los problemas de conducta exhibidos por los alumnos; problemas de salud de los docentes; la falta de apoyo de parte de los padres y madres de familia. Todas estas situaciones pueden estar vinculadas a que la motivación externa de los maestros del centro escolar esté afectada en forma desfavorable.

Si bien la motivación extrínseca está determinada por muchos factores, ello no impide que tanto el personal docente, el equipo de dirección que lidera y gerencia la escuela, como la población estudiantil expresan que la labor docente se realiza con un buen grado de motivación, como para demostrar relaciones sanas de convivencia, con percepción estudiantil de docentes comprometidos con su misión educativa e interesados en continuar mejorando su trabajo.

A partir de los sustentos anteriores se puede decir que el impacto de factores externos, si bien se han vivido y han sido expresados, no dejan ver un deterioro en cuanto a la motivación extrínseca de docentes, lo cual hasta podría llegar a considerarse como una labor que contribuye en la zona a la prevención de violencia juvenil, en tanto la población joven que asiste a clases, se estimula ante la energía creativa del quehacer de sus docentes. 
Los párrafos anteriores indican que los maestros, además de conservar motivación laboral docente, saben identificar con propiedad aquellas situaciones que complican su ambiente de trabajo. Y es por ello, precisamente, que tienen una opinión de peso en cuanto a proponer qué medidas se deban realizar para estimular la motivación docente. Es por ello que el equipo de investigadores del estudio citado consideró necesario estructurar sus propuestas, las cuales se detallan con mayor amplitud en el siguiente apartado.

\section{Medidas que incidan en el fortalecimiento de la motivación intrínseca y extrínseca de los docentes frente a la violencia juvenil}

Al ser consultado el personal docente acerca de medidas que fortalezcan la motivación laboral docente, expresaron concretamente cinco ideas: a) la asignación de los recursos pedagógicos necesarios al docente, como elemento que les permitiría mejorar el trabajo que realizan y contribuir al sentimiento de satisfacción laboral; b) la necesidad de innovar con capacitaciones que les permitan mantener y no perder su vinculación con el aprendizaje en términos más amplios; c) trabajo en equipo en la escuela; d) espacios de esparcimiento (deportes, charlas, actividades sociales, talleres vocacionales), y e) generar espacios para abordar el tema de la violencia compartiendo experiencias, puntos de vista, etcétera.

La consulta con los jóvenes, por su parte, se divide en tres medidas que podrían prevenir la violencia y fomentar la motivación del docente: a) mejorar las relaciones entre compañeros; b) proyectos que fomenten las habilidades sociales y resolución de problemas, y c) mejorar el ambiente de trabajo del docente.

Tanto el personal de dirección como el docente creen en la importancia de que la familia puede ser un punto de apoyo en la motivación laboral y en la prevención de la violencia juvenil en la escuela; por lo que es necesario un rol más activo por parte de las familias para la educación y el fomento de valores en los jóvenes. 


\section{Discusión final}

Con todo lo expuesto, se puede afirmar que el Centro Escolar República Oriental del Uruguay posee un equipo docente que cuenta con un punto de apoyo fundamental en su misión educativa: la resiliencia. Este factor ha dotado a estos maestros de la fortaleza necesaria en medio del escenario difícil en el que desempeñan su labor educativa.

El término resiliencia viene del latín "resilio" que quiere decir rebotar o recuperar su forma original, en sus términos más generales supone ser un proceso dinámico y ofrece "la capacidad humana de enfrentar, sobreponerse y ser fortalecido o transformado por experiencias de la adversidad" (Grotberg, en Ayala, Fabián y Najarro, 2012).

Para Rutter (en Sobarzo, 2011), la resiliencia se compone por un conjunto de procesos sociales e intrapsíquicos que posibilitan tener una vida "sana" en un medio "insano". Dichos procesos tendrían lugar a través del tiempo, que no son producto del desarrollo humano, sino que se trataría de un proceso que caracteriza un complejo sistema social en un momento determinado.

La resiliencia es, pues, la capacidad humana de enfrentar factores negativos como el estrés, adversidad o contexto negativo obteniendo como resultado una adaptación positiva, posibilitando un equilibrio físico y mental que permiten obtener una mejor calidad de vida en las personas (Martínez, Portillo y Portillo, 2014) .

Picardo (2012) caracteriza la resiliencia docente en los siguientes términos. En el ámbito escolar, los docentes resilientes son capaces de implicarse en un proyecto educativo compartido con el resto de la comunidad, que buscan expresamente el desarrollo de todos los alumnos.

La capacidad de ocuparse de la formación de sujetos inmaduros, dependientes, necesitados de muchas cosas, de niños afectados por experiencias adversas supone que los maestros son suficientemente resilientes, es decir, emocionalmente estables, con alta motivación de logro, buena tolerancia a la frustración, de espíritu animoso e identificados con su trabajo.

El profesor resiliente construye resiliencia en los alumnos cuando los acepta y aprecia tal cual son, al margen de su ren- 
dimiento académico. El contacto afectuoso, expresado física o verbalmente, es de gran importancia para establecer y mantener vínculos positivos con las personas que nos importan.

Los resultados de la investigación citada denotan que la motivación laboral ha permitido a los docentes a seguir adelante y hacer frente a la violencia manifiesta dentro y fuera de las escuelas $\mathrm{y}$ a las carencias materiales, de insumos y de recursos que caracterizan a la escuela salvadoreña. Aunado a ello, el docente también es resiliente. Lo anterior ofrece una situación de fortaleza y oportunidad que debe ser capitalizada para abordar la temática.

El riesgo de "abandonar" al docente en la escuela podría traer consecuencias que perjudicarían los resultados positivos que los alumnos obtienen de sus maestros. Es decir, la relación que existe entre el profesor y el alumno podría verse perjudicada en tanto que el interés del primero hacia el alumno podría perderse, lo mismo que esa cercanía que posibilita la confianza dentro y fuera del salón, con lo que los alumnos podrían perder a un aliado o a esa persona que "cree en ellos", y los maestros podrían verse incomodados en mayor medida, con las manifestaciones de violencia.

Desde luego que para lograr un salto cualitativo en la escuela, se deben resolver otras problemáticas en las que está sumergida la realidad salvadoreña: delincuencia/inseguridad, economía, desempleo, violencia/pandillas, corrupción, etc. (IUDOP, 2016). Sin embargo, y con mucha más razón, es necesario que mientras el docente siga sobrellevando esta situación de desventaja en la escuela, se le formará en su dimensión humana, enfatizando fortalecer esas potencialidades y recursos personales para hacer frente a la adversidad, para desarrollar competencias y construir en circunstancias adversas y estresantes de manera sostenida.

De acuerdo al Observatorio del Ministerio de Educación de El Salvador (MINED) de 2015 sobre los centros educativos públicos de El Salvador, $82.20 \%$ de los docentes ha recibido formación. Las temáticas que en las que los docentes se han formado son las siguientes: escuela inclusiva de tiempo pleno y sistemas integrados, protección y seguridad, educación sexual, ley de protección integral de la niñez y la adolescencia (conocida como LEPINA), educación inclusiva, educación (en asignaturas de matemáticas, física, química, lenguaje, ciencias sociales, inglés, etc.) y otros sin especificar. 
Dentro del Plan Nacional de Formación de Docentes en Servicio en el Sector Público, según el MINED, a finales de 2015, se formaron 20290 maestros y maestras, cifra que equivale a casi la mitad de la planta docente nacional. Este grupo incluye a 7436 docentes de I y II ciclos (matemática, lenguaje, ciencias naturales y ciencias sociales), 3571 docentes de III ciclo y media (química, física, biología, lenguaje, sociales y matemática), así como 300 especialistas de inglés, 300 especialistas de Educación inicial y parvularia y 300 especialistas de Arte, cultura, recreación y Deportes. Se incluye, además, la formación de 8383 docentes en Tecnologías de la información y comunicación (TIC) para uso pedagógico, lo cual contribuye a la implementación del Programa presidencial una niña, un nińo, una computadora (MINED, 2015c).

Se puede apreciar que, entre los temas impartidos en las formacón docente, no hay alguno en que se refiera a formación humana orientada a la capacidad de trabajar en el aula en condiciones adversas. En caso de incluirse, se reforzarían contenidos tales como el empoderamiento, autoestima, asertividad, resolución de conflictos, acciones prosociales (solidaridad, cooperación y altruismo), control emocional, percepción de autoeficacia, seguridad y confianza, locus de control, etc. Con ello se podrían fortalecer elementos tales como la vocación profesional, la admiración por el papel que el docente juega dentro de la sociedad, el valor de la educación para el país, lo gratificante que resulta ser docente, la importancia de su protagonismo para el alumno y lo necesario del acompañamiento de este en el proceso de desarrollo de competencias y en el desarrollo socioafectivo de sus alumnos.

Ese imperativo categórico del docente, esa vocación profesional y ese compromiso por la docencia debe ser capitalizado por las autoridades, ya que en la medida que se robustezca, el docente tendrá la capacidad de seguir en esa actitud positiva de trabajar, a pesar que las condiciones que el centro escolar en cuanto a violencia y a otras carencias. Es preciso, pues, desarrollar la motivación y la resiliencia en el docente; esto posibilitaría crear un maestro optimista, con la convicción que, incluso en las condiciones actuales de la escuela, se pueden obtener resultados positivos en el alumno. 
Esos rasgos de motivación y de resiliencia del docente, hasta el momento invisibilizados, han dotado a la escuela de una fortaleza que le ha permitido seguir adelante en condiciones adversas, mientras que, a la vez, se convierten una oportunidad, en cuanto que el docente se ha convertido en un actor fundamental en la prevención de la violencia en los entornos escolares, así como en auténticos agentes de cambio en la sociedad salvadoreña.

\section{Referencias bibliográficas}

Ayala, M., Cruz, M., Novoa, A. Satisfacción laboral y motivación docente de instituciones educativas públicas y privadas. Antiguo Cuscatlán. Tesis no publicada, Universidad Centroamericana José Simeón Cañas, UCA, 2011.

Ayala, R., Fabián, C., Najarro, L. Factores protectores de riesgo y fuentes de resiliencia en mujeres lisiadas de guerra de la Asociación de Lisiados de Guerra, ALGES, Antiguo Cuscatlán.Tesis no publicada, Universidad Centroamericana José Simeón Cañas, UCA, 2012.

Amaya, L., Martínez, J. "Sureños en El Salvador. Un acercamiento antropológico a las pandillas de deportados", en Revista Realidad y Reflexión, Universidad Francisco Gavidia, año 14, núm. 39, enero-julio, 2014. Disponible en: http:// www.ufg.edu.sv/icti/doc/icti-RyR39.pdf

Bolaños, H. SIMEDUCO: 38 estudiantes y 10 maestros muertos en 2014, en La Prensa Gráfica, 10 de diciembre de 2014. Disponible en: http://www.laprensagrafica.com/ 2014/12/10/simeduco-38-estudiantes-y-10-maestrosasesinados-en-2014

Borgonovo, L., López, G., Rodríguez, K. Estudio sobre rendimiento escolar y la motivación laboral de docentes. Antiguo Cuscatlán. Tesis no publicada, Universidad Centroamericana José Simeón Cañas, UCA, 2007.

Cruz, M., Perla, C. Factores personales y profesionales relacionados con la motivación de los docentes. Antiguo Cuscatlán. Tesis no publicada, Universidad Centroamericana José Simeón Cañas, UCA, 2009. 
Feixas, M. "La influencia de factores personales, institucionales y contextuales en la trayectoria y el desarrollo docente de los profesores universitarios", en Revista Educar, Universitat Autónoma de Barcelona, núm. 33, 2004, pp 31-59. Disponible en: http://ddd.uab.cat/pub/educar/0211819Xn33p31.pdf

Flores, M, Mejía, C., Muñoz, A. "Impacto de la violencia juvenil en la motivación hacia el trabajo de docentes del tercer ciclo de educación básica en el centro escolar República Oriental del Uruguay en Mejicanos: un estudio de caso". Antiguo Cuscatlán. Tesis no publicada, Universidad Centroamericana José Simeón Cañas, UCA, 2014.

Flores, R. "SIMEDUCO: 12 docentes asesinados en 2015", en $\mathrm{La}$ Prensa Gráfica, 2 de septiembre de 2015. Disponible en: http://www.laprensagrafica.com/2015/09/02/simeduco12-docentes-asesinados-en-2015

Guzmán, J. y Monterrosa, G. "La prevención del acoso escolar (bullying) a través de la formación inicial y en servicio de los docentes que laboran en los centros educativos públicos en alto riesgo social”, Antiguo Cuscatlán. Tesis no publicada, Universidad Centroamericana José Simeón Cañas, UCA, 2013.

Hernández, W. "Siete profesores asesinados en el 2016", en ELSALVADOR.COM, 22 de junio de 2016. Disponible en: http://www.elsalvador.com/articulo/sucesos/siete-profesores-asesinados-2016-116717.

IUDOP. "Los salvadoreños evalúan el segundo año de gobierno de Sánchez Cerén", en Boletín de prensa, año XXX, núm. 2, 2016. Disponible en: http://www.uca.edu.sv/iudop/ wpcontent/uploads/INFORME_EVAGOB16.pdf

Martínez, G., Portillo, C., Portillo, K. Resiliencia en estudiantes universitarios de nuevo ingreso. Antiguo Cuscatlán. Tesis no publicada, Universidad Centroamericana José Simeón Cañas, UCA. 2014.

MINED. Ejes estratégicos del Plan Nacional de Educación en Función de la Nación, San Salvador, El Salvador, MINED. 2015 a.

MINED. Observatorio MINED 2015 sobre los centros educativos públicos de El Salvador, San Salvador, MINED, 2015 b. 
MINED. Formación Docente, El Salvador, MINED, 2015c. Disaponible en red: https://www.mined.gob.sv/index.php/ programas-educativos/formacion-docente

Organización Mundial de la Salud. Asamblea Mundial de la Salud, 1996.

Picardo, O. "Resiliencia: hacer educación en entornos desfavorecidos", en Revista Realidad y Reflexión, núm. 34, Universidad Francisco Gavidia, 2012, pp. 47-65. Disponible en: http://www.ufg.edu.sv/icti/doc/RyRN34-Picardo.pdf

Sobarzo, J. Taller de Fomento de la resiliencia, 2011. Disponible en:http://es.slideshare.net/anadahi/taller-de-fomento-dela-resiliencia. 